\title{
The Human Hippocampus: Cognitive Maps or Relational Memory?
}

\author{
Dharshan Kumaran and Eleanor A. Maguire \\ Wellcome Department of Imaging Neuroscience, Institute of Neurology, University College London, London WC1N 3BG, United Kingdom
}

The hippocampus is widely accepted to play a pivotal role in memory. Two influential theories offer competing accounts of its fundamental operating mechanism. The cognitive map theory posits a special role in mapping large-scale space, whereas the relational theory argues it supports amodal relational processing. Here, we pit the two theories against each other using a novel paradigm in which the relational processing involved in navigating in a city was matched with similar navigational and relational processing demands in a nonspatial (social) domain. During functional magnetic resonance imaging, participants determined the optimal route either between friends' homes or between the friends themselves using social connections. Separate brain networks were engaged preferentially during the two tasks, with hippocampal activation driven only by spatial relational processing. We conclude that the human hippocampus appears to have a bias toward the processing of spatial relationships, in accordance with the cognitive map theory. Our results both advance our understanding of the nature of the hippocampal contribution to memory and provide insights into how social networks are instantiated at the neural level.

Key words: fMRI; hippocampus; memory; relational; cognitive map; spatial cognition

\section{Introduction}

Navigation in large-scale space is widely acknowledged to involve the hippocampus (for review, see Burgess et al., 2002). Consequently, the cognitive map theory, arising from the discovery of location-specific firing of place cells in the rodent hippocampus, posits that a fundamental function of the hippocampus is the construction and maintenance of spatial maps of the environment (O'Keefe and Nadel, 1978). However, this view is disputed. The relational theory suggests the hippocampus processes all manner of associations and sequences of events that comprise our daily lives, linking these into relational frameworks (Cohen and Eichenbaum, 1993; Eichenbaum, 2004). According to this perspective, the hippocampus mediates large-scale relational frameworks for, among others, family trees and city layouts, which allow us to make novel detours through our city or make inferences about the relationships between members of our family (Eichenbaum, 2000). Thus, spatial navigation is proposed to be merely one example of the way in which the hippocampus is specialized for amodal "relational processing" (Eichenbaum et al., 1999).

It is important to note that the term relational processing has been used in the literature to describe different cognitive operations, ranging from the binding together of multiple constituents

Received March 21, 2005; revised June 23, 2005; accepted June 26, 2005.

This work was supported by a Wellcome Trust senior research fellowship in basic biomedical science to E.A.M. We thank C. Frith and J. $0^{\prime}$ Keefe for helpful discussions and 0 . David for technical advice. We declare that we have no competing financial interests.

Correspondence should be addressed to Dharshan Kumaran, Wellcome Department of Imaging Neuroscience, Institute of Neurology, University College London, 12 Queen Square, London WC1N 3BG, UK. E-mail: d.kumaran@fil.ion.ucl.ac.uk or e.maguire@fil.ion.ucl.ac.uk.

D0I:10.1523/JNEUROSCI.1103-05.2005

Copyright $\odot 2005$ Society for Neuroscience $\quad$ 0270-6474/05/257254-06\$15.00/0 of scenes or events into long-term memory to the flexible expression of memories (Cohen et al., 1999; Eichenbaum et al., 1999; Davachi and Wagner, 2002; Ryan and Cohen, 2004). Here, we use relational processing to refer to flexible memory expression whereby inferential judgments and indirect associations are made about information acquired across many different episodes stored within a so-called "memory space" (Eichenbaum et al., 1999). Although there is some evidence to suggest that the human hippocampus may be specifically involved in processing simple associations and relationships (for review, see Eichenbaum, 2004), its proposed role in supporting large-scale relational frameworks of the kind described in the relational theory has not, until now, been investigated.

We took as our starting point the spatial task that is generally agreed to engage the hippocampus in both rodents and humans, namely navigation in a large-scale environment (in this case, the Greater London area, UK). Our aim was to design an analogous task, placing similar demands on navigation and relational processing but within a nonspatial domain. Just as navigation through space is a fundamental part of our lives, we each operate in a social domain in which we negotiate our network of friends and acquaintances. At first glance, remembering one's social network appears markedly different from remembering the layout of one's local environment. In fact, there are a number of striking parallels between the two memory representations. Both can be conceptualized mathematically as consisting of nodes (landmarks or people) and edges (e.g., roads or social connections), forming a highly interconnected graph or network (Lynch, 1960; Wasserman and Faust, 1994). Importantly, one can "navigate" through either, entering and exiting at any given point (node), choosing the optimal route based on current priorities and circumstances. 
We used functional magnetic resonance imaging (fMRI) to compare patterns of brain activation while subjects navigated within either a spatial domain (their city) or a nonspatial domain (their social network). According to the relational account, the hippocampus should be engaged by both tasks to a similar degree, regardless of domain. In contrast, the cognitive map theory implies that hippocampal activation should be driven primarily by relational processing within a spatial, but not a nonspatial (social), domain. Our principal aim, therefore, was to distinguish between these two influential theories and in doing so further our understanding of the fundamental operating mechanism of the human hippocampus.

\section{Materials and Methods}

Participants. Eighteen healthy right-handed native English speakers participated (nine males; mean age, 24 years; SD, 2.84). All had a similar background (current or recent university students) and had been resident in London for at least 1 year (average, 16.6 years; SD, 8.5). Inclusion criteria for the experiment were that a subject had at least 14 friends living in Greater London whose homes they had visited and that the friends formed a connected social network (see Fig. $1 A$ ). In addition, we ensured a range (from a few months to over a decade) in temporal durations for both acquaintance and location knowledge to prevent age of memories influencing results. The average clustering coefficient (Borgatti et al., 1999 ) of participants' social network was 0.66 (SD, 0.11). All subjects gave informed written consent in accordance with the local research ethics committee.

Stimuli. Participants were interviewed at least 2 weeks before scanning (average, 4.8 weeks; SD, 2.0). They provided details of their friends, including a rating ( 5 point scale) of how well their friends knew each other $(0=$ two people had never met through $4=$ they knew each other very well). A score of 2 implied the pair of people knew each other. For each participant, the same set of 14 friends formed the basis for all experimental conditions. Stimuli for experimental tasks were drawn from this basic set of 14 friends and comprised eight first names. If two friends shared a first name, the initial of their surnames was also presented. Names were displayed in white text on a black background and arranged on the screen as a regularly spaced array (Fig. $1 B$ ). The same array was never repeated. As far as possible, names appeared with equal frequency in each of the four experimental tasks. In the low-level baseline task, high-frequency unfamiliar names identified by the subject in the initial interview were used. These were approximately matched for length and number of syllables to names of the subject's friends.

Tasks. Instructions for the relational tasks were as follows: person A (at the top left of the array) has a crate of wine that is to be given to person $B$ (at the top right). In the social relational task, a person must know another person to give the crate to him/her. In the spatial task, however, the person receiving the crate must live physically closer (as the crow flies) to person B. In both conditions, each time a person receives the crate, a bottle of wine is removed, the task being to determine the maximum number of bottles of wine that can be removed from the crate before person $B$ receives it $(0,1,2,3$, or more). In the nonrelational tasks, the subjects were instructed to proceed systematically through the array of names, constructing and focusing on a detailed mental image of each of their friends' faces (social) or the outside of their residences (spatial). They were required to determine how many friends either wore glasses (social) or lived in a specific category of building (spatial). In the lowlevel baseline task, subjects were told to read each name silently and determine the number of names with exactly two vowels. Pilot studies before the scanning experiment suggested that on average subjects required $15 \mathrm{~s}$ to complete the relational (spatial, $14.7 \mathrm{~s}, \mathrm{SD}, 4.0$; social, $15.7 \mathrm{~s}, \mathrm{SD}, 1.7$ ) and nonrelational tasks (spatial, $15.1 \mathrm{~s}, \mathrm{SD}, 1.6$; social, $14.5 \mathrm{~s}, \mathrm{SD}, 0.7)$. However, initial piloting in the scanning environment revealed that subjects required $\sim 20 \mathrm{~s}$ to comfortably perform the tasks. Before scanning, participants had extensive practice to familiarize them with the tasks. Scanning consisted of two main sessions (each comprised five trials of each task pseudorandomly intermixed) followed by a shorter third session (see below). Each trial began with a condition-specific cue displayed for $5 \mathrm{~s}($ Fig. $1 \mathrm{~B})$ : wine/visit, wine/know, building/picture, glasses/picture, vowel/read. The array of names was then displayed for $20 \mathrm{~s}$, during which subjects performed the relevant task. Subjects made their response, not during the $20 \mathrm{~s}$ while the array was displayed but in a subsequent $5 \mathrm{~s}$ response period. Responses for all tasks were made via a four-button keypad. Tasks were designed to be equated for numbers of each response type (i.e., 0, 1, 2, 3, or more). There was a variable rest period (6-9s) before the next trial. After the two main sessions, subjects participated in a shorter third session during which they were instructed to perform the tasks as they had been in the main sessions but with one difference. In this third session, subjects were required to respond as soon as the task was complete instead of in a subsequent response period. The purpose of this session was to verify the time spent on each task (see Table 1). After scanning, in a debriefing session, subjects were asked to rate each task along a number of parameters (see Table 1) and to produce a simple sketch map of where their 14 friends live. Subjects also reported that they thought only about friends in the array and did not think back to the initial interview. Spatial relational questions were scored according to either the map drawn in the debriefing session or a real map of London. An error in distance calculation of $15 \%$ was permitted. In an analogous way, questions in the social relational task were scored according to either the initial social network (prescan interview) or the network elicited (in an identical way) in the postscan debriefing session. The nonrelational tasks were scored based on factual information previously provided by subjects.

Neuroimaging. T2-weighted echo planar (EPI) images with blood oxygen level-dependent contrast were acquired on a 1.5 tesla Siemens AG (Erlangen, Germany) Sonata MRI scanner. We used standard scanning parameters to achieve whole-brain coverage: 44 slices, $2 \mathrm{~mm}$ thickness (1 $\mathrm{mm}$ gap), repetition time of $3.96 \mathrm{~s}$. The first $6 \mathrm{vol}$ from each session were discarded to allow for T1 equilibration effects. A T1-weighted structural MRI scan was acquired for each subject. During scanning, eye position was monitored using an infrared tracker. Images were analyzed in a standard manner using the statistical parametric mapping software SPM2 (www.fil.ion.ucl.ac.uk/spm). Spatial preprocessing consisted of realignment, normalization to a standard EPI template in Montreal Neurological Institute space with a resampled voxel size of $3 \times 3 \times 3 \mathrm{~mm}$, and smoothing using a Gaussian kernel with full width at half maximum of 8 $\mathrm{mm}$. After preprocessing, statistical analysis was performed using the general linear model. The experiment had a two-by-two factorial design, the factors being task (relational or nonrelational) and domain (spatial or social). Our interest was in the 20 s period when subjects were viewing the arrays and performing the tasks. This period was modeled as a boxcar function (of $20 \mathrm{~s}$ duration) and convolved with the canonical hemodynamic response function to create regressors of interest. Subject-specific movement parameters were included as regressors of no interest. Subject-specific parameter estimates pertaining to each regressor (betas) were calculated for each voxel. These parameter estimates (collapsed across sessions) were entered into a second level random-effects analysis using a one-way ANOVA. We report results in a priori regions of interest [previously identified in neuroimaging studies of spatial (Burgess et al., 2002; Hartley et al., 2003) and social (Adolphs, 2003; Gallagher and Frith, 2003; Rilling et al., 2004; Singer et al., 2004) cognition] at $p<0.001$, uncorrected for multiple comparisons, with an extent threshold of $>6$ contiguous voxels. Activations in other regions are reported if they survive whole-brain correction for multiple comparisons at $p<0.05$.

\section{Results}

\section{Behavioral data}

Subjects were debriefed thoroughly after scanning to assess how well the tasks were matched on a range of important variables such as task difficulty, task success, and the types of cognitive processes engaged. The behavioral results are reported in Table 1 and clearly show that the only significant difference between the two relational tasks was, as we predicted, the frequency of thoughts evoked about how people knew one another and where they lived in relationship to each other. Knowing thoughts were more frequent in the social relational task (maximum rating 
Table 1. Scores on behavioral measures

\begin{tabular}{|c|c|c|c|c|c|}
\hline Parameter & Social R & Spatial R & Social NR & Spatial NR & Baseline \\
\hline Performance & $74(13.8)$ & $80(15.0)$ & $91(9.0)$ & $90(11.4)$ & $96(8.1)$ \\
\hline Difficulty & $6.0(1.7)$ & $6.3(2.0)$ & $3.7(1.7)$ & $3.9(1.5)$ & $2.1(1.4)$ \\
\hline Reaction time (s) & $15.8(4.3)$ & $16.3(4.0)$ & $18.6(2.0)$ & $18.7(2.0)$ & $17.0(3.6)$ \\
\hline Time on task & $1.6(0.7)$ & $1.4(0.6)$ & $2.2(0.7)$ & $2.1(0.6)$ & $2.6(0.8)$ \\
\hline Knowing & $5(0)$ & $1.6(1.2)$ & $0.4(0.7)$ & $0.3(0.6)$ & $\mathrm{n} / \mathrm{a}$ \\
\hline Location & $1.3(1.1)$ & $5(0)$ & $0.1(0.2)$ & $1.9(1.2)$ & $\mathrm{n} / \mathrm{a}$ \\
\hline Episodic memory & $3.0(1.2)$ & $2.3(1.3)$ & $2.4(1.7)$ & $2.3(1.6)$ & $\mathrm{n} / \mathrm{a}$ \\
\hline Emotional & $1.3(1.5)$ & $0.9(0.9)$ & $1.7(1.6)$ & $0.8(1.0)$ & $\mathrm{n} / \mathrm{a}$ \\
\hline Self-related & $2.5(1.3)$ & $2.3(1.7)$ & $0.9(1.0)$ & $1.3(1.1)$ & $\mathrm{n} / \mathrm{a}$ \\
\hline Imagery & $2.5(1.5)$ & $3(1.7)$ & $5(0)$ & $5(0)$ & $\mathrm{n} / \mathrm{a}$ \\
\hline
\end{tabular}

SDs are in parentheses. $R$, Relational; NR, nonrelational; $n / a$, not applicable. Performance is a percentage score. Difficulty was rated out of 10 , where $10=$ maximal difficulty. Time on task was rated as follows: $0=$ much too little time; $1=$ just too little; $2=$ about right; $3=$ bit too much time; $4=$ much too much time. For other parameters, the question was "how often did you have these type of thoughts?": $0=$ never; $1=$ very rarely; $2=$ rarely; $3=$ once every few trials; $4=$ once per trial; $5=$ more than once per trial. Reaction times were obtained from the third session that followed the two main scanning sessions (see Materials and Methods).

given by all participants; $\left.t_{(17)}=11.8 ; p<0.0001\right)$, and location thoughts were more frequent in the spatial relational task (again maximum rating given by all participants; $t_{(17)}=-14.3 ; p<$ 0.0001). In all other respects, the spatial and social relational tasks were comparable, as indeed were the two nonrelational tasks. This is also true of the reaction times, with the data from the third session in accordance with previous pilot data suggesting that $20 \mathrm{~s}$ was optimal for task completion and also showing that the two relational tasks took similar times to complete. Given the pairs of relational and nonrelational tasks were so well matched behaviorally, we next examined the fMRI data to see whether they were similarly comparable at the neural level.

\section{Neuroimaging data}

Separate sets of brain regions were engaged preferentially during spatial and social navigation. There was significantly greater activation during the spatial relational task relative to the social relational task within a well defined network that comprised the following brain regions (peak coordinate; $x, y, z$ ) (Fig. 1C): right posterior hippocampus, extending throughout the posterior and into the body $(30,-36,-3 ; z=3.95)$, left posterior hippocampus $(-33,-42,-6 ; z=5.52)$, right parahippocampal gyrus (27, $-33,-15 ; z=5.91)$, left parahippocampal gyrus $(-30,-39,-9$; $z=5.10)$, right retrosplenial cortex $(15,-57,15 ; z=7.30)$, left retrosplenial cortex $(-18,-60,15 ; z=6.53)$, right posterior parietal cortex $(42,-78,39 ; z=5.43)$, and left posterior parietal cortex $(-39,-81,30 ; z=4.97)$.

In marked contrast, a separate set of brain regions was significantly more active in the social relational compared with the spatial relational task (Fig. 1D). This comprised the left dorsomedial prefrontal cortex $(-3,48,39 ; z=5.09)$, ventromedial prefrontal cortex $(-6,48,3, z=4.45 ;-3,51,-21, z=4.58)$, left and right lateral orbitofrontal cortex $(-33,24,-24, z=4.33 ; 33$, $24,-24, z=3.54)$, left inferior frontal gyrus $(42,30,-6 ; z=$ $5.09)$, left anterior insula $(-30,15,-3 ; z=4.09)$, left mid superior temporal sulcus (STS) $(-66,-18,-9 ; z=5.41)$, left posterior STS $(-57,-51,12 ; z=3.60)$, left temporoparietal junction $(-48,-51,24 ; z=3.84)$, left and right temporal poles $(-48,12$, $-36, z=3.89 ; 45,21,-36, z=4.18)$, left posterior cingulate cortex $(-6,-51,30 ; z=3.94)$, right lingual gyrus $(9,-78,-3$; $z=4.15)$, left middle occipital gyrus $(-36,-66,-3 ; z=4.03)$, left fusiform gyrus $(-42,-60,-21 ; z=3.25)$, and right cerebel$\operatorname{lum}(27,-87,-33 ; z=4.17)$. No significant medial temporal lobe activation was associated with the social relational task, even when compared with the low level baseline vowel counting task (see Materials and Methods). Moreover, no significant hippocampal activation was observed in this contrast even when a more liberal statistical threshold ( $p<0.01$ uncorrected) was used. This contrasts with significant bilateral hippocampal activation observed in the comparison of the spatial relational task with the low-level baseline vowel counting task $(p<0.001$, uncorrected).

These results show that the hippocampus was active when subjects performed a task requiring relational processing in a spatial, but not a nonspatial (social), domain. We next examined the contrast pertaining to the main effect of task. Here, we observed significantly greater activation in the relational tasks compared with the nonrelational tasks independent of domain within a distributed set of brain regions (supplemental Fig. 1, available at www.jneurosci.org as supplemental material), consistent with the greater demands imposed by the relational tasks on cognitive processes such as working memory and the allocation of attentional resources (Corbetta and Shulman, 2002). Importantly, no hippocampal activation was evident in this contrast (the main effect of task) or when we performed an additional analysis looking for regions engaged in common during the relational tasks compared with the nonrelational tasks, using a conservative masking procedure (supplemental Fig. 2, available at www. jneurosci.org as supplemental material). Thus, these results provide additional support for the notion that relational processing per se does not drive hippocampal activation in these tasks.

An obvious question that arises is whether spatial processing of a more general nature accounts for hippocampal activation in the spatial relational task. To answer this, we directly compared the two nonrelational tasks. As with the relational tasks, right parahippocampal gyrus $(27,-39,-15 ; z=5.34)$, left parahippocampal gyrus $(-30,-45,-12 ; z=6.41)$, right retrosplenial cortex $(15,-57,21 ; z=5.94)$, left retrosplenial cortex $(-15$, $-63,21 ; z=5.13)$, right posterior parietal cortex $(45,-72,33$; $z=5.22)$, and left posterior parietal cortex $(-42,-81,30 ; z=$ 5.82 ) were more active in the spatial compared with the social nonrelational task. Therefore, it would seem that these brain areas are concerned with the broader aspects of spatial processing, consistent with previous studies involving visuo-spatial stimuli (Epstein and Kanwisher, 1998; Maguire et al., 1998a; Burgess et al., 2002). That the hippocampus was not observed to be active in this contrast suggests its primary role is in spatial relational processing as exemplified by mental navigation within London.

Our findings suggest that neither relational nor spatial processing alone is sufficient to drive the hippocampus but that the combination of the two factors is crucial to its engagement. Given that we used a factorial design, we were able to statistically assess 
A

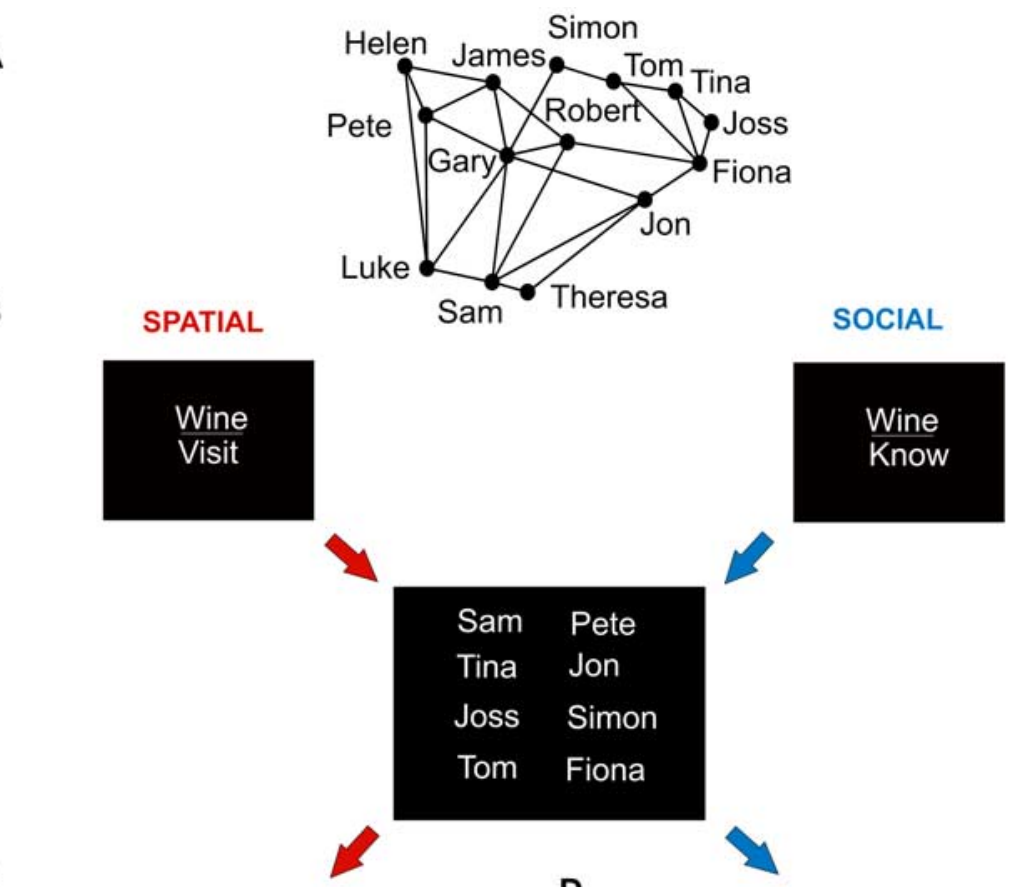

C
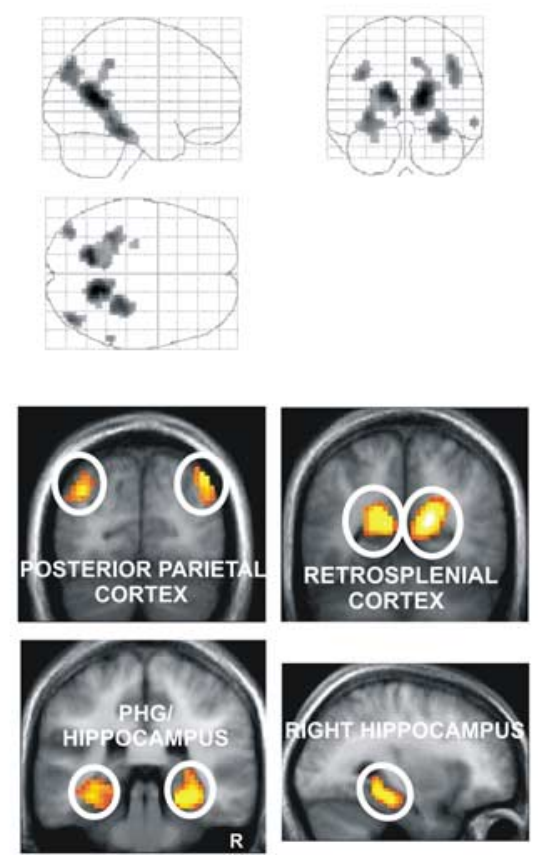

D
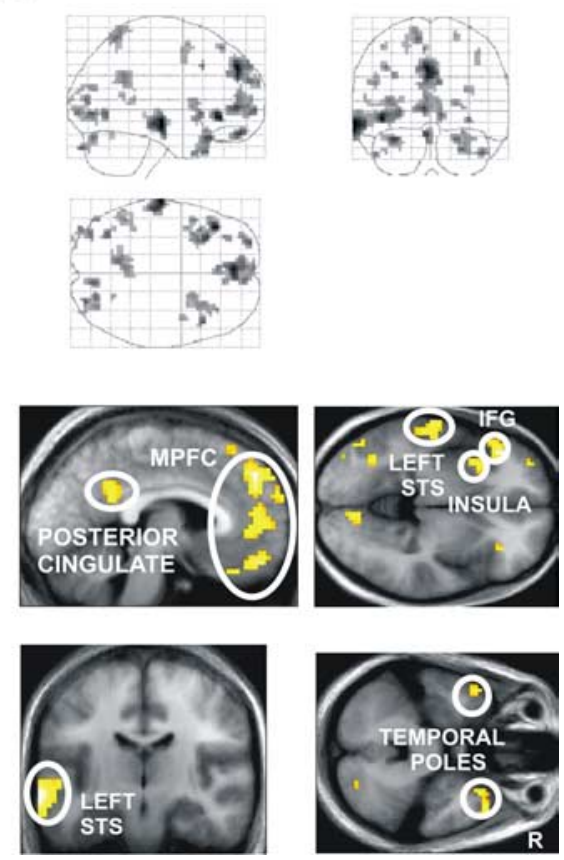

Figure 1. Example stimulus and results of relational task comparisons. $A$, Schematic representation (never seen by subjects) of one subject's network of friends. Lines between names indicate two people know each other (see Materials and Methods). The spatial arrangement of names represents their relative locations in London. $\boldsymbol{B}$, Cues for relational tasks and an example stimulus array. C, Brain areas significantly more active when subjects mentally navigated within London (see Results). PHG, Parahippocampal gyrus. D, Brain areas significantly more active when subjects navigated their social network (see Results). MPFC, Medial prefrontal cortex; IFG, inferior frontal gyrus. "Glass brain" figures (displayed above). Activations shown on the averaged structural MRI scan of the 18 participants (displayed below). R, Right side of the brain. Colors correspond to $z$-scores (orange/yellow/white in increasing order). The threshold is set at $p<0.001$, uncorrected.

the effect of one factor (domain) on the other (task) by examining the interaction (Fig. 2). Significant effects were found in only one brain region, namely the right (and to a lesser extent left) hippocampus (peak coordinate, $30,-36,-3 ; z=4.28$ ), confirming differential activation in the spatial versus the nonspatial (social) domain during relational compared with nonrelational task performance (Fig. 2). Interestingly, the reverse interaction permitted additional probing of the social brain network and revealed the following areas were driven by the combination of social and relational task performance: left mid STS $(-66,-18,-9 ; z=4.98)$, posterior cingulate cortex $(-6,-39,24 ; z=3.69)$, right temporoparietal junction $(60,-57,21$; $z=3.45)$, and left posterior STS $(-57$, $-51,12 ; z=3.78)$.

\section{Discussion}

In this study, we used functional brain imaging to address a long-standing debate concerning the fundamental operating mechanism of the human hippocampus. We compared patterns of brain activation while subjects performed two tasks placing similar demands on relational processing: navigation within either a spatial domain (their city) or a nonspatial domain (their social network). We show that execution of these two complex tasks resulted in very different patterns of brain activation, although the two tasks were closely matched at the behavioral level. Crucially, the hippocampus was only engaged by relational processing in a spatial, but not a nonspatial (social), domain. The present results not only advance our understanding of how the hippocampus contributes to memory but also provide the first insights into how social networks are instantiated at the neural level.

When subjects navigated within their city, hippocampus together with parahippocampal, retrosplenial, and posterior parietal cortices were preferentially engaged. These findings are highly consistent with previous neuroimaging studies of spatial navigation (Ghaem et al., 1997; Maguire et al., 1997, 1998b; Burgess et al., 2002; Hartley et al., 2003). They provide confirmatory evidence that this network of brain regions is involved in guiding us through large-scale space and that the participants were genuinely engaged in mental navigation within London. Furthermore, our results are in agreement with previous work suggesting that these individual brain areas make distinct contributions to the overall process of spatial navigation (Burgess et al., 2002). We show that parahippocampal, retrosplenial, and posterior parietal cortices were engaged by spatial processing per se, regardless of the task performed. In contrast, the hippocampus was only engaged when subjects were required to focus on the spatial relationships between places (their friends' houses) as they navigated through their city but not when they thought of the places themselves. Thus, these results provide additional support for the notion that the hippocampus forms the cornerstone of a distributed network that 

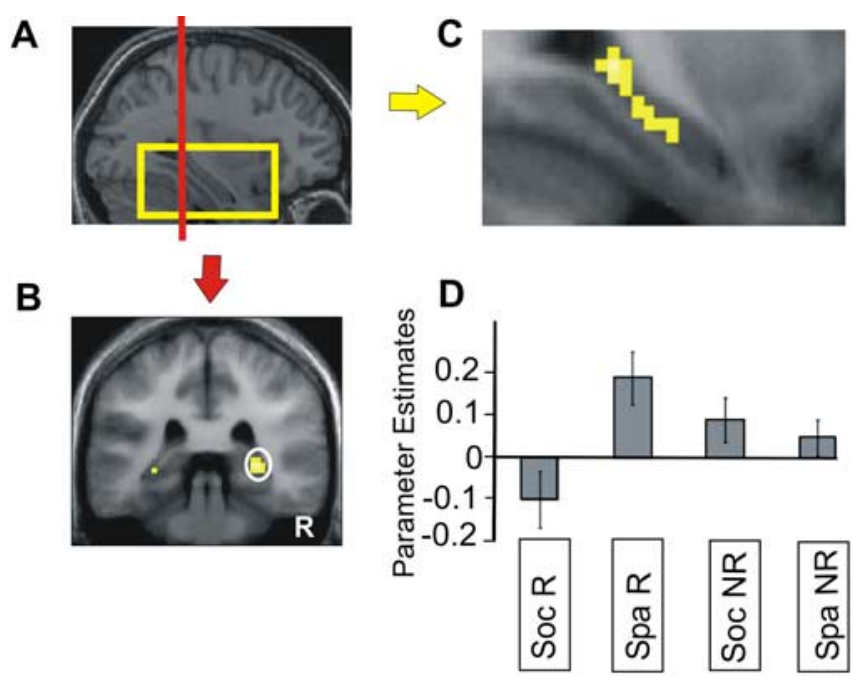

Figure 2. Interaction effect of domain and task. $\boldsymbol{A}$, Sagittal section from the structural MRI scan of one subject chosen at random. The yellow box outlines the region displayed in $C$. The red line indicates the location of the coronal section in the next panel. The threshold is set at $p<$ 0.001 , uncorrected. $\boldsymbol{B}$, Coronal section at the level of the peak in right posterior hippocampus. Smaller left hippocampal activation is also evident. R, Right side of the brain. C, Sagittal view showing the extent of activation in the posterior and body of the right hippocampus. $\boldsymbol{D}$, Condition-specific parameter estimates (betas) in arbitrary units averaged across subjects, relative to the baseline task at a representative voxel in the center of cluster in right hippocampus. Bars represent the SE. Soc R, Social relational; Spa R, spatial relational; Soc NR, social nonrelational; Spa NR, spatial nonrelational.

supports spatial navigation in humans through its adeptness at processing the complex relationships between locations in our environment.

In contrast, when subjects navigated within their social network, a separate set of brain regions was significantly more active. This consisted of several brain areas including medial prefrontal cortex, insula, STS, posterior cingulate cortex, temporoparietal junction, and temporal poles, which all form key components of the proposed neural circuitry of social cognition (Adolphs, 2003). The exact contribution of each brain area to performance of this complex, multifaceted task remains an open question. Navigation through one's social network involves several different cognitive processes. These include the retrieval of knowledge about both the friends themselves and their relationship to one another, as well as the adoption of another's viewpoint, or mentalizing. Thus, one possibility is that the ability to mentalize is crucial in allowing us to navigate efficiently through our social network and that this process involves the STS, medial prefrontal cortex, temporal poles, temporoparietal junction, and posterior cingulate cortex, regions activated previously in functional imaging studies of theory of mind (Allison et al., 2000; Gallagher et al., 2000; Adolphs, 2003; Gallagher and Frith, 2003; Saxe and Kanwisher, 2003; Rilling et al., 2004).

Our primary aim in this study was to distinguish between two competing theories of hippocampal function to advance our understanding of the underlying mechanisms by which the human hippocampus supports memory. The cognitive map theory holds that the hippocampus preferentially processes the spatial relationships between locations in the environment, and it is this that is critical to its role in memory across species (O'Keefe and Nadel, 1978). In contrast, the relational theory argues that the hippocampus does not differentiate between spatial and nonspatial information but is instead specialized for relational processing (Cohen and Eichenbaum, 1993). Evidence from electrophysio- logical and lesion studies suggest that the rodent hippocampus may be involved not only in the performance of spatial tasks (Morris et al., 1982) but also some nonspatial tasks (for review, see Eichenbaum et al., 1999, 2004). However, several studies have suggested that rats' performance on spatial tasks such as the Morris water maze may be particularly sensitive to hippocampal damage (O’Keefe, 1999; Burton et al., 2000; Gilbert and Kesner, 2002; Broadbent et al., 2004; Jarrard et al., 2004). Thus, the question as to whether the rodent hippocampus has a bias toward the processing of spatial relationships has not been definitively answered.

In humans, previous studies provide some support for the notion that the hippocampus processes simple associations between items such as words and faces (for review, see Eichenbaum, 2004; Squire et al., 2004). Several fMRI studies have demonstrated that the human hippocampus may also be involved in processing the relationships between overlapping pairs of stimuli and thus mediate inferential (transitive) judgments on novel stimulus pairs (Heckers et al., 2004; Preston et al., 2004). However, none of these studies has investigated the role of the hippocampus in supporting large-scale relational memory representations that underlie flexible memory expression, as described in the relational theory. Furthermore, previous neuroimaging studies have not addressed the question of whether the hippocampus is equally involved in relational processing within both spatial and nonspatial domains. The current study speaks directly to these issues, because subjects performed two tasks with "real world" relevance, both placing similar demands on relational processing and requiring flexible use of relational memory representations but differing in terms of domain being navigated (spatial or nonspatial). Thus, we were able to directly compare spatial navigation with a nonspatial task similar in terms of scale (i.e., multiple associations interlinked in a large relational framework) and ecological validity.

Our results show that separate brain networks were preferentially engaged during the spatial and social relational tasks, with hippocampal activation only observed during the spatial relational task. One explanation for the clear differences in patterns of brain activation observed during the two relational tasks is a mismatch between the two tasks across factors such as overall task difficulty or the frequency of autobiographical memories elicited. However, given the two relational tasks were so well matched across eight relevant task parameters (Table 1), we believe this to be unlikely. Thus, as we predicted, the two relational tasks only differed with respect to the frequency of thoughts evoked about how people knew one another and where they lived in relationship to each other. Furthermore, the same set of friends formed the basis for both relational tasks, making it unlikely that differences in stimulus novelty contributed to the observed pattern of results.

Another possibility is that, despite our best efforts, the two relational tasks might have differed in terms of relational processing required. Indeed, it has been predicted that the amount of relational processing required during a memory task will modulate hippocampal engagement (Cohen et al., 1999). In both relational tasks, subjects were required to use relational memory representations in a flexible way to successfully navigate between nodes (friends or places) within their network. Thus, both tasks placed substantial demands on relational processing. Moreover, that the two relational tasks were comparable in terms of task difficulty and time on task argues against an appreciable difference in relational processing demands. However, despite all the evidence that the two relational tasks were as closely matched as 
possible, one cannot be categorical that the two tasks were wholly equivalent in terms of relational processing demands, given the difficulty in quantifying relational processing and the complex nature of the tasks. In this respect, our failure to observe hippocampal activation in the social relational task even when we compared it to the low-level baseline vowel counting task is insightful. This observation seems to indicate that relational processing alone may not be sufficient to drive hippocampal engagement. Instead, our results suggest that it is the combination of spatial and relational processing as exemplified by mental navigation through London that is crucial to hippocampal engagement.

There are many diverse views concerning the nature of the hippocampal contribution to memory. In this study, we focus on two prominent theories that occupy opposing positions in their evaluation of how exactly the hippocampus supports memory. Our results, in showing that it is the combination of spatial and relational processing that drives hippocampal engagement, provide evidence in favor of the cognitive map theory of hippocampal function (O'Keefe and Nadel, 1978). Thus, relational processing per se may not be the fundamental operating mechanism of the human hippocampus, at least when the relevant memory frameworks are established and in continuous everyday use. However, the view that the human hippocampus acts primarily to create and store representations of the physical environment (O'Keefe and Nadel, 1978) must be interpreted within the context of a wealth of evidence both from neuropsychological analyses of amnesic patients and neuroimaging studies on healthy subjects, suggesting that the human hippocampus plays a role not only in spatial memory but in many other aspects of memory (Scoville and Milner, 1957) (for review, see Squire et al., 2004). Although the exact contribution of the human hippocampus to familiarity-based recognition and semantic memory is still widely debated, there is a general consensus it is critical for episodic memory in humans (Scoville and Milner, 1957; Tulving, 2002; Squire et al., 2004). Whether the spatial mnemonic functions of the hippocampus also underlie its role in episodic memory, our capacity to recollect personally experienced events set in a unique spatiotemporal context, remains to be determined. Thus, although our results suggest that the human hippocampus has a bias toward the processing of spatial relationships, additional work is needed to explore how this relates to the more pervasive role of the hippocampus in many aspects of memory.

\section{References}

Adolphs R (2003) Cognitive neuroscience of human social behaviour. Nat Rev Neurosci 4:165-178.

Allison T, Puce A, McCarthy G (2000) Social perception from visual cues: role of the STS region. Trends Cogn Sci 4:267-278.

Borgatti SP, Everett MG, Freeman LC (1999) UCINET 6.0 version 1.00. Harvard, MA: Analytic Technologies.

Broadbent NJ, Squire LR, Clark RE (2004) Spatial memory, recognition memory, and the hippocampus. Proc Natl Acad Sci USA 101:14515-14520.

Burgess N, Maguire EA, O'Keefe J (2002) The human hippocampus and spatial and episodic memory. Neuron 35:625-641.

Burton S, Murphy D, Qureshi U, Sutton P, O’Keefe J (2000) Combined lesions of hippocampus and subiculum do not produce deficits in a nonspatial social olfactory memory task. J Neurosci 20:5468-5475.

Cohen NJ, Eichenbaum H (1993) Memory, amnesia and the hippocampal system. Cambridge, MA: MIT.

Cohen NJ, Ryan J, Hunt C, Romine L, Wszalek T, Nash C (1999) Hippocampal system and declarative (relational) memory: summarizing the data from functional neuroimaging studies. Hippocampus 9:83-98.
Corbetta M, Shulman GL (2002) Control of goal-directed and stimulusdriven attention in the brain. Nat Rev Neurosci 3:201-215.

Davachi L, Wagner AD (2002) Hippocampal contributions to episodic encoding: insights from relational and item-based learning. J Neurophysiol 88:982-990.

Eichenbaum H (2000) A cortical-hippocampal system for declarative memory. Nat Rev Neurosci 1:41-50.

Eichenbaum H (2004) Hippocampus: cognitive processes and neural representations that underlie declarative memory. Neuron 44:109-120.

Eichenbaum H, Dudchenko P, Wood E, Shapiro M, Tanila H (1999) The hippocampus, memory, and place cells: is it spatial memory or a memory space? Neuron 23:209-226.

Epstein R, Kanwisher N (1998) A cortical representation of the local visual environment. Nature 392:598-601.

Gallagher HL, Frith CD (2003) Functional imaging of "theory of mind." Trends Cogn Sci 7:77-83.

Gallagher HL, Happe F, Brunswick N, Fletcher PC, Frith U, Frith CD (2000) Reading the mind in cartoons and stories: an fMRI study of 'theory of mind' in verbal and nonverbal tasks. Neuropsychologia 38:11-21.

Ghaem O, Mellet E, Crivello F, Tzourio N, Mazoyer B, Berthoz A, Denis M (1997) Mental navigation along memorized routes activates the hippocampus, precuneus, and insula. NeuroReport 8:739-744.

Gilbert PE, Kesner RP (2002) Role of the rodent hippocampus in pairedassociate learning involving associations between a stimulus and a spatial location. Behav Neurosci 116:63-71.

Hartley T, Maguire EA, Spiers HJ, Burgess N (2003) The well-worn route and the path less travelled: distinct neural bases of route following and wayfinding in humans. Neuron 37:877-888.

Heckers S, Zalesak M, Weiss AP, Ditman T, Titone D (2004) Hippocampal activation during transitive inference in humans. Hippocampus 14:153-162.

Jarrard LE, Davidson TL, Bowring B (2004) Functional differentiation within the medial temporal lobe in the rat. Hippocampus 14:434-449.

Lynch K (1960) The image of the city, pp 46-90. Cambridge, MA: MIT.

Maguire EA, Frackowiak RS, Frith CD (1997) Recalling routes around London: activation of the right hippocampus in taxi drivers. J Neurosci 17:7103-7110.

Maguire EA, Frith CD, Burgess N, Donnett JG, O’Keefe J (1998a) Knowing where things are parahippocampal involvement in encoding object locations in virtual large-scale space. J Cogn Neurosci 10:61-76.

Maguire EA, Burgess N, Donnett JG, Frackowiak RS, Frith CD, O'Keefe J (1998b) Knowing where and getting there: a human navigation network. Science 280:921-924.

Morris RGM, Garrud P, Rawlins JN, O'Keefe J (1982) Place navigation impaired in rats with hippocampal lesions. Nature 297:681-683.

O'Keefe J (1999) Do hippocampal pyramidal cells signal non-spatial as well as spatial information? Hippocampus 9:352-364.

O’Keefe J, Nadel L (1978) The hippocampus as a cognitive map. Oxford: Oxford UP

Preston AR, Shrager Y, Dudukovic NM, Gabrieli JD (2004) Hippocampal contribution to the novel use of relational information in declarative memory. Hippocampus 14:148-152.

Rilling JK, Sanfey AG, Aronson JA, Nystrom LE, Cohen JD (2004) The neural correlates of theory of mind within interpersonal interactions. NeuroImage 22:1694-1703.

Ryan JD, Cohen NJ (2004) Processing and short-term retention of relational information in amnesia. Neuropsychologia 42:497-511.

Saxe R, Kanwisher N (2003) People thinking about thinking people. The role of the temporo-parietal junction in "theory of mind." NeuroImage 19:1835-1842.

Scoville WB, Milner B (1957) Loss of recent memory after bilateral hippocampal lesions. J Neurol Neurosurg Psychiatry 20:11-12.

Singer T, Kiebel SJ, Winston JS, Dolan RJ, Frith CD (2004) Brain responses to the acquired moral status of faces. Neuron 41:653-662.

Squire LR, Stark CE, Clark RE (2004) The medial temporal lobe. Annu Rev Neurosci 27:279-306.

Tulving E (2002) Episodic memory: from mind to brain. Annu Rev Psychol 53:1-25.

Wasserman S, Faust K (1994) Social network analysis: methods and applications. Cambridge, UK: Cambridge UP. 